\title{
Acquired noncaustic esophageal strictures in children
}

\author{
Elif Sag, MD', Aysenur Bahadir, MD², Mustafa Imamoglu, MD², Sefa Sag, MD', Gokce Pinar Reis, MD², Erol Erduran, MD², \\ Murat Cakir, MD ${ }^{1}$ \\ ${ }^{1}$ Department of Pediatric Gastroenterology Hepatology and Nutrition, Faculty of Medicine, Karadeniz Technical University, Trabzon, Turkey; ${ }^{2}$ Department of Pediatric \\ Hematology and Oncology, Faculty of Medicine, Karadeniz Technical University, Trabzon, Turkey; ${ }^{3}$ Department of Pediatric Surgery, Faculty of Medicine, Karadeniz \\ Technical University, Trabzon, Turkey; ${ }^{4}$ Department of Pediatric Surgery, Kanuni Education and Research Hospital, Trabzon, Turkey
}

Background: Esophageal stricture (ES) is an uncommon clinic entity in pediatrics that may be congenital or acquired in childhood. Acquired noncaustic ES is very rare, and clinical features of affected patients are unknown.

Purpose: We aimed to evaluate the clinical findings, and outcomes of patients with acquired noncaustic ES to aid physicians in the early referral of patients to gastroenterologists.

Methods: The medical data of patients with acquired noncaustic ES who were followed in our gastroenterology clinic between January 2009 and December 2019 were reviewed.

Results: Acquired noncaustic ES was found in 12 of the 4,950 patients $(0.24 \%)$ who underwent endoscopy during the study period. The main symptoms were dysphagia (58.3\%), vomiting (33.3\%), and chronic anemia (8.3\%). Chronic malnutrition and underweight were found in $66.6 \%$ of the patients. The most common etiological factors were radiotherapy, peptic reflux, and achalasia (16.6\%, each), while chemotherapy, squamous-cell carcinoma (SC) of the esophagus, eosinophilic esophagitis (EoE), esophageal web, epidermolysis bullosa, and esophageal diverticulum $(8.2 \%$, each) were the other etiological factors. Patients with EoE underwent endoscopic bougie dilation in addition to steroid use and elimination diet. Patients with epidermolysis bullosa and esophageal web underwent bougie dilation. Patients with peptic reflux-related ES were initially put on antireflux therapy, but during follow-up, one patient required esophageal replacement with colonic interposition. Patients with radiotherapy-related ES recovered with medical therapy. The patient with initially underwent surgical gastrostomy and tumoral mass excision. The patient then received chemotherapy and radiotherapy and underwent jejunal interposition. Patients with achalasia underwent surgical esophagomyotomy.

Conclusion: The presence of solid dysphagia, malnutrition, and an associated disease may alert physicians to the presence of ES.

Key words: Dysphagia, Esophageal stricture, Malnutrition

\begin{abstract}
Key message
Question: Which clinical findings suggest esophageal structure in children with dysphagia?

Finding: The presence of solid dysphagia, malnutrition, and a comorbid condition is suggestive of esophageal stricture in children with dysphagia.

Meaning: Patients with findings suggestive of noncaustic esophageal stricture should receive early referral to pediatric gastroenterology units.
\end{abstract}

\section{Introduction}

Esophageal stricture (ES) is an uncommon clinic entity in pediatrics. It may be congenital (10\%-15\%) or acquired in childhood. ${ }^{1)}$ The most common types of congenital ES are congenital webs, stenosis due to bronchial remnants and idiopathic congenital muscular hypertrophy of esophagus. Most of them are presented in the early infancy and most cases require surgical intervention. ${ }^{2)}$ Acquired ES are mainly related due to caustic ingestions $(50 \%-60 \%)$ or postsurgical anastomotic stenosis $(10 \%-20 \%)$. These patients are mainly admitted to pediatric surgery departments, and management, treatment algorithms and follow-up of these patients are well-defined in the literature.,3) Inflammatory $(<1 \%)$, peptic $(3 \%-4 \%)$, or tumoral $(<0.1 \%)$ stenosis of the esophagus are very rare and consist of noncaustic or noncorrosive ES in childhood. ${ }^{2)}$ These patients are generally referred to pediatric gastroenterology clinics for the suspected esophageal or peptic problem and diagnosed after endoscopic or radiological examination. Early diagnosis of these patients is essential because prolonged feeding problems may cause nutritional deficiencies and aspiration pneumonia.

In this study, we aimed to analyze the demographic and clinical parameters and outcome of the children with acquired noncaustic ES in our center in order to pay attention physician for early referral of these patients to a pediatric gastroenterology center. 


\section{Methods}

Medical data of patients with acquired noncaustic ES (esophageal narrowing) who were followed in our pediatric gastroenterology clinic between January 2009 and December 2019 were analyzed from the hospital file records retrospectively. Data including demographic characteristics, etiological factors, and clinical, endoscopic and radiological findings, treatment modality and the outcomes of the patients were recorded. The patients with corrosive, congenital and postsurgical anastomotic ES were not included into the study.

A written informed consent was obtained from each patient. The study protocol was approved by the institutional ethics committee (study number: 2018/262). Statistical analysis was performed using the SPSS ver. 16.0 (SPSS Inc., Chicago, IL, USA). Continuous variables were expressed in mean \pm standard deviation (SD) and categorical variables were expressed in number $(\mathrm{n})$ and percentage (\%).

\section{Results}

Acquired noncaustic $E S$ was found in 12 ( 6 females; mean \pm SD age, $10.1 \pm 4.6$ years; range, $2-16.5$ years) of the 4,950 patients $(0.24 \%$; $95 \%$ confidence interval, $0.1 \%-0.4 \%)$ who were performed endoscopy during the study period (Table 1$)$.

The main symptoms of the patients were dysphagia $(n=7$, $58.3 \%)$ followed by vomiting $(\mathrm{n}=4,33.3 \%)$ and chronic anemia $(n=1,8.3 \%)$. Dysphagia was associated with solids in 6 of 7 patients while 1 patient was associated with liquids and solids (patient X). Duration of the symptoms was ranged from 10 days to 180 months (median, 2 months), and 3 patients (25\%) were received antiacid/proton-pump inhibitor (PPI) treatment for their symptoms previously. Both frequency of chronic malnutrition (height $\mathrm{z}$ score $<-2 \mathrm{SD}$ ) and underweight (weight $\mathrm{z}$ score $<-2 \mathrm{SD}$ ) was found in $66.6 \%$.

Endoscopic and radiological examinations revealed mideso- phageal strictures in 6 patients (50\%), distal ES in 4 patients (33.3\%), and proximal ES in 2 patients $(16.6 \%)$. The most common etiological factors were radiotherapy $(n=2,16.6 \%$, Ewing's sarcoma [30 Gray to spinal vertebra for 10 days] and acute lymphoblastic leukemia [ALL, 18 Gray to spinal vertebra for 12 days]), secondary to GERD ( $n=2,16.6 \%$, Cornelia De Lange $[\mathrm{CDL}]$ syndrome and cerebral palsy) and achalasia $(\mathrm{n}=2$, $16.6 \%)$, while chemotherapy ( $\mathrm{n}=1,8.3 \%$, ALL-Down syndrome, St Jude total XV ALL - 20th maintenance therapy), squamous-cell carcinoma of the esophagus (ESCC, $n=1,8.3 \%$ ), eosinophilic esophagitis (EoE) $(n=1,8.3 \%)$, esophageal web (Plummer-Vinson syndrome [PVS], $\mathrm{n}=1,8.3 \%$ ), epidermolysis bullosa $(\mathrm{n}=1,8.3 \%)$ and esophageal diverticulum (ED, $\mathrm{n}=1$, 8.2\%) were the other etiological factors (Fig. 1).

Patient with EoE underwent endoscopic bougie dilation 2 sessions in addition to systemic and local steroids $(2 \mathrm{mg} / \mathrm{kg}$ for 2 weeks, tapered and fluticasone propionate was initiated $880 \mu \mathrm{g}$ in the first 3 months followed by a lower dose of $440 \mu \mathrm{g}$ for 9 months), PPI ( $2 \mathrm{mg} / \mathrm{kg}$ ) and 6 food elimination diet. Patient with epidermolysis bullosa and PVS underwent one session endoscopic bougie dilation. Patients with GERD related ES $(n=2)$ were initially put on a medical antireflux therapy (PPI, $2 \mathrm{mg} / \mathrm{kg}$ ). The symptoms of the patient with cerebral palsy resolved with medical therapy; however, surgical gastrostomy was performed in the other patient with CDL syndrome. On the follow-up, patient with CDL syndrome was suffered from increased oral secretion and respiratory problems due to chronic aspiration. Then, esophageal replacement with colonic interposition was performed. Patients with radiotherapy-related ES $(n=2)$ recovered with medical therapy for 3 months (PPI, $2 \mathrm{mg} / \mathrm{kg}$ and sucralfate, $20 \mathrm{mg} / \mathrm{kg}$ ). The patient with ESCC initially underwent surgical gastrostomy and tumoral mass excision. Then, the patient was received chemotherapy (paclitaxel+cisplatin) and radiotherapy for the primary tumor and underwent jejunal interposition 10 months later. Patients with achalasia $(n=2)$ underwent surgical esophagomyotomy.

During a mean follow-up of $30.3 \pm 11.1$ months, no compli-

Table 1. Patients' demographic characteristics and clinical findings

\begin{tabular}{|c|c|c|c|c|c|c|}
\hline Patient & Age $(y r) / s e x$ & Weight $z$ score & Height $z$ score & Major symptom & Duration of symptom & $\begin{array}{l}\text { Previous antiacid/ } \\
\text { PPI treatment }\end{array}$ \\
\hline I & $5 / M$ & -2.26 & -3.84 & Solid dysphagia & 1 Month & No \\
\hline$\|$ & $16 / \mathrm{M}$ & -3.45 & -2.03 & Solid dysphagia & 10 Days & No \\
\hline III & $8 / F$ & -3.57 & -5.58 & Vomiting & 1 Month & Yes \\
\hline IV & $10 / F$ & -2.10 & -0.4 & Solid dysphagia & 2 Months & No \\
\hline V & $12 / F$ & -1.58 & 0.07 & Vomiting & 2 Months & No \\
\hline $\mathrm{VI}$ & $5.5 / F$ & -3.95 & -6.3 & Vomiting & 1 Month & Yes \\
\hline VII & $12 / F$ & -2.24 & -2.81 & Vomiting & 1 Month & No \\
\hline VIII & $7.5 / \mathrm{M}$ & -0.77 & 0.35 & Solid dysphagia & 6 Months & Yes \\
\hline IX & 2/M & 0.09 & 0.5 & Solid dysphagia & 15 Days & No \\
\hline$x$ & $15 / M$ & -3.83 & -2.01 & Solid and liquid dysphagia & 3 Months & No \\
\hline$X I$ & $16.5 / F$ & -0.1 & -2.15 & Chronic anemia & 18 Months & No \\
\hline$X \|$ & 13/M & -3.78 & -3.01 & Dysphagia & 1 Month & No \\
\hline
\end{tabular}

PPI, proton-pump inhibitor. 


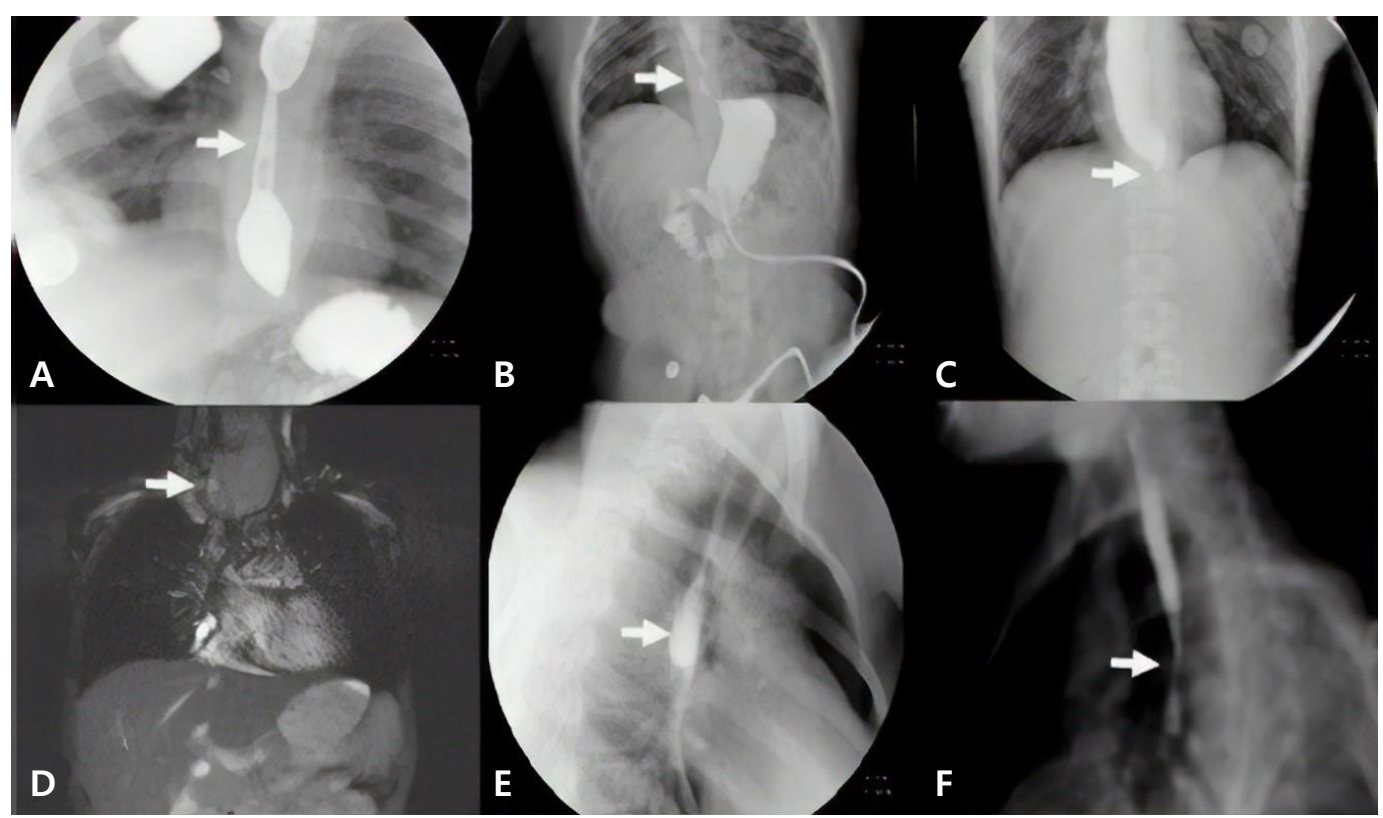

Fig. 1. (A) A barium swallow study showing a midesophageal stricture in a patient after chemotherapy (patient I) (white arrow). (B) A barium swallow study of a patient with CDL syndrome and ES (distal) secondary to GERD (patient VI) (white arrow). (C) A barium swallow study showing distal narrowing in a patient with achalasia (patient VII) (white arrow). (D) Magnetic resonance imaging of a patient with ESCC showing proximal ES (patient X) (white arrow). (E) A barium swallow study showing midesophageal diverticulum (patient IV) (white arrow). (F) A barium swallow study showing midnarrowing in a patient with epidermolysis bullosa (patient XII) (white arrow). CDL, Cornelia De Lange; ES, esophageal stricture; ESCC, squamous-cell carcinoma of the esophagus; GERD, gastroesophageal reflux disease.

Table 2. Etiology, concomitant conditions, treatment modalities, and outcome of patients with esophageal stricture

\begin{tabular}{|c|c|c|c|c|c|}
\hline Patient & Etiology & $\begin{array}{l}\text { Associated } \\
\text { disease }\end{array}$ & $\begin{array}{l}\text { Localization } \\
\text { of ES }\end{array}$ & Treatment & Duration of follow-up (mo)/outcome \\
\hline I & Chemotherapy & ALL, DS & Mid & Bougie dilation & 34/Asymptomatic \\
\hline$\|$ & Radiotherapy & Ewing sarcoma & Mid & PPI, Sucralfate & 25/Asymptomatic \\
\hline III & GERD & MMR, epilepsy & Distal & PPI & 29/Exist dysphagia for solid food \\
\hline IV & ED & - & Mid & PPI & 27/Asymptomatic \\
\hline V & Achalasia & - & Distal & Esophagomyotomy & 25/Asymptomatic \\
\hline $\mathrm{VI}$ & GERD & CDL syndrome & Distal & Gastrostomy, Colonic interposition & 32/Asymptomatic \\
\hline VII & Achalasia & - & Distal & Esophagomyotomy & 33/Asymptomatic \\
\hline VIII & Inflammation (EoE) & Atopy & Mid & CS, Bougie dilation, elimination diet & 33/Asymptomatic with cow's milk elimination \\
\hline IX & Radiotherapy & ALL & Mid & PPI, sucralfate & 23/Asymptomatic \\
\hline$x$ & Tumoral mass & ESCC & Proximal & Chemoradiotherapy, Jejunal interposition & 64/Asymptomatic \\
\hline$X I$ & Esophageal web & PVS & Proximal & Bougie dilation & 21/Asymptomatic \\
\hline XII & Mucosal adherence & EB & Mid & Bougie dilation & 18/Asymptomatic \\
\hline
\end{tabular}

ES, esophageal stricture; ALL, acute lymphoblastic leukemia; DS, Down syndrome; PPI, proton-pump inhibitor; GERD, gastroesophageal reflux disease; MMR, mental and motor retardation; ED, esophageal diverticulum; CDL, Cornelia De Lange; EoE, eosinophilic esophagitis; CS, corticosteroids; ESCC, squamous-cell carcinoma of the esophagus; PVS, Plummer-Vinson syndrome; EB, epidermolysis bullosa.

cation or relapse was observed in the patients who were operated due to achalasia and the other patients undergoing bougie dilatation. However, patient with ESCC underwent bougie dilation for 3 sessions due to anastomotic stricture. The patient with CDL syndrome was also followed with gastrostomy and the patient with cerebral palsy was followed only with a PPI and standard polymeric formula. The patient with EoE is only under cow's milk elimination diet and the patient with ED was treated by a PPI $2 \mathrm{mg} / \mathrm{kg}$ during the symptomatic period (Table 2). Nutrition status of the patients with malnutrition was improved in all patients (except patient III and XII)

\section{Discussion}

In this study, we share our experience about the acquired noncaustic ES in children. We found that (1) esophageal dysphagia for solids and vomiting are the major symptoms for the noncaustic ES in children, (2) most of the patient had malnutrition at the time of admission, (3) median time between the symptoms and diagnosis was 2 months, (4) noncaustic ES in children is a consequence of wide range of clinical conditions such as complications of anticancer treatment, peptic stricture, achalasia, esophageal tumors and EoE, and (5) outcome of the patients 
depends on the underlying diseases.

Apart from caustic injuries, esophageal dysphagia is usually the result of either structural (strictures, rings, webs) or inflammatory abnormalities (reflux or EoE) or motility disorders (achalasia or diffuse esophageal spasm). ${ }^{5}$ Malign esophageal tumors are extremely rare in childhood. Patients initially admitted with acute onset liquid dysphagia, and then solid dysphagia may develop in untreated patients due to esophageal stenosis. Only patients with early achalasia may be initially admitted with solid dysphagia. Patients with symptoms of esophageal dysphagia should be assessed by history for alarm symptoms such as presence of both solid and liquid dysphagia, and malnutrition that suggests esophageal stenosis. Early endoscopic and radiological examination is essential in patients with alarm symptoms. A PPI trial is suggested in children without alarm symptoms. If dysphagia persists after a 4-week PPI trial, further investigations by endoscopic examination are essential. Once inflammatory or structural lesions have been ruled out by endoscopy (and histopathology), barium swallow or esophageal manometry if available should be used to evaluate all children with persistent dysphagia. ${ }^{5}$ Additionally, presence of an associated disease such as neurological squeal, under anticancer treatment or EB increases likelihood of strictures in patients with esophageal dysphagia. Apart from dysphagia, patients with ES may be admitted with chronic vomiting. Sometimes, chronic iron deficiency anemia may be the only findings in early period in patients with PVS.

In this study, we found that acquired noncaustic ES was mainly located in middle esophagus. Although, we could not find any association between the localization and underlying etiology due to small number of patients, stricture is located distally in reflux esophagitis and achalasia, and proximally in PVS.

Esophageal strictures due to anticancer treatment in pediatric age are very rare. The main risk for the ES is radiotherapy to chest and neck region. It is a dose-related side-effect and associated with progressive obliterative endarteritis and ischemia on the esophageal mucosa which leads the fibrosis. It affects $1 \%$ of the patients undergoing radiotherapy. ${ }^{6}$ Additionally, chemotherapeutic agents such as doxorubicin, cyclophosphamide, bleomy. cin, fluorouracil, dactinomycin, cisplatin, methotrexate and etoposide, and infections such as candidiasis may also be associated with ES in patients receiving anticancer treatment. ${ }^{7}$ In an extensive review published recently, 130 pediatric patients with ALL were followed for 3 years and the incidence of ES was found to be $3 \%$ ( $n=2$ candida esophagitis and $n=2$ associated with chemotherapy). ${ }^{8)}$ We believe that we will encounter with the ES much more in the future in the early or late period due to increased use of aggressive treatment protocols and survival rate these patients.

Peptic ESs are the one of the major complications of chronic reflux esophagitis. Stricture formation occurs in $7 \%-23 \%$ of patients with reflux esophagitis. ${ }^{9)}$ It occurs usually at the squamocolumnar junction and measures $1-4 \mathrm{~cm}$ in length. Significant predictors of stricture formation in patients with GERD are lower esophageal sphincter tone of $<8 \mathrm{mmHg}$, impaired esophageal motility (due to neurological problems) and duodenogastric reflux. Hiatus hernia is twice as prevalent in GERD patients with stricture (85\%) when compared to those with no esophagitis. ${ }^{10,11)}$

Symptomatic ES should be dilated, either using balloons or bouginage. Corticosteroids may be used in patients with EoE. PPIs may be useful in patients with mild ES associated with peptic reflux. In case of refractory ES, mytomycin-C, diathermy, laser and/or stenting can be useful. It is important to minimize the need for surgery and esophageal replacement. ${ }^{1)}$

In conclusion, we share our experience about ES in children who were admitted to pediatric gastroenterology department in order to pay attention to general physician for the early referral of these patients. Presence of solid dysphagia, malnutrition and an associated disease may alarm for the presence of ES.

Conflicts of interest

No potential conflict of interest relevant to this article was reported.

\section{References}

1. Vandenplas Y. Management of benign esophageal strictures in children. Pediatr Gastroenterol Hepatol Nutr 2017;20:211-5.

2. Aprigliano F. Chevalier Jackson Lecture. Esophageal stenosis in children. Ann Otol Rhinol Laryngol 1980;89:391-6.

3. Doğan Y, Erkan T, Cokuğraş FC, Kutlu T. Caustic gastroesophageal lesions in childhood: an analysis of 473 cases. Clin Pediatr (Phila) 2006;45:4358.

4. Baskin D, Urganci N, Abbasoğlu L, Alkim C, Yalçin M, Karadağ C, et al. A standardised protocol for the acute management of corrosive ingestion in children. Pediatr Surg Int 2004;20:824-8.

5. Sanghavi R, Rosen R. Esophageal dysphagia. In: Ongkasuwan J, Chiou $\mathrm{EH}$, editors. Pediatric dysphagia: challenges and controversies. Cham: Springer, 2018:215-38.

6. Vanagunas A, Jacob P, Olinger E. Radiation-induced esophageal injury: a spectrum from esophagitis to cancer. Am J Gastroenterol 1990;85:80812.

7. Ellenhorn JD, Lambroza A, Lindsley KL, LaQuaglia MP. Treatmentrelated esophageal stricture in pediatric patients with cancer. Cancer 1993;71:4084-90.

8. Kelly K, Storey L, O' Sullivan M, Butler K, McDermott M, Corbally M, et al. Esophageal strictures during treatment for acute lymphoblastic leukemia. J Pediatr Hematol Oncol 2010;32:124-7.

9. Achem SR, Devault KR. Dysphagia in aging. J Clin Gastroenterol 2005; 39:357-71.

10. Zouari M, Kamoun H, Bouthour H, Ben Abdallah R, Hlel Y, Ben Malek $\mathrm{R}$, et al. Peptic oesophageal stricture in children: management problems. Afr J Paediatr Surg 2014;11:22-5.

11. Pearson EG, Downey EC, Barnhart DC, Scaife ER, Rollins MD, Black $\mathrm{RE}$, et al. Reflux esophageal stricture--a review of 30 years' experience in children. J Pediatr Surg 2010;45:2356-60.

How to cite this article: Sag E, Bahadir A, Imamoglu M, Sag S, Reis GP, Erduran E, et al. Acquired noncaustic esophageal strictures in children. Clin Exp Pediatr 2020;63:447-50. https:// doi.org/10.3345/cep.2020.00199 